\title{
Multiple Listeria Abscesses in an Immunocompetent Patient
}

\author{
Heitor C. Frade ${ }^{1}$, Chandra Pingili ${ }^{1}$, Premkumar Nattanamai ${ }^{1}$ \\ 1. Neurology, University of Missouri, Columbia, USA
}

Corresponding author: Heitor C. Frade, heitorfrade@gmail.com

\begin{abstract}
Listeria monocytogenes is a ubiquitous organism that can potentially cause gastroenteritis and, less commonly, central nervous system infections. Brain abscess is rare and often associated with immunocompromised status. We report a case of multiple abscesses caused by Listeria in a previously immunocompetent elderly patient who developed a headache and left-sided hemiparesis over the course of days. Neuroimaging studies revealed multiple ring-enhancing lesions in the brain and midbrain territories. Blood culture, brain tissue aspirate, and cerebrospinal fluid nucleic acid amplification test were positive for Listeria. Extensive immunologic workup revealed no primary or secondary immunodeficiency disorders. After the initiation of antibiotics, the patient showed gradual clinical improvement and went to a skilled nursing facility after two weeks.
\end{abstract}

Categories: Neurology, Infectious Disease

Keywords: listeria monocytogenes, brain abscess, central nervous system infections

\section{Introduction}

Listeria monocytogenes is a foodborne pathogen that most commonly causes gastroenteritis but may also affect the central nervous system (CNS), particularly in newborns, pregnant women, elderly and immunosuppressed patients. It is estimated that 1,600 new cases of listeriosis occur every year in the United States [1].

Listeria reach the CNS via hematogenous spread by crossing blood-brain and blood-choroid barriers. Although the most common CNS manifestation is meningitis, which accounts for $20 \%$ of bacterial meningeal infections in both neonates and elderly patients, it may also present as encephalitis, rhombencephalitis, and brain abscess $[2,3]$.

Brain abscesses account for less than $10 \%$ of CNS listerial infections. They are mostly singular lesions, located on cortical or subcortical regions. When symptomatic, common findings include fever, mental status changes and focal neurological deficits. Bacteremia is often present, in comparison to other bacterial brain abscesses, while cerebrospinal fluid (CSF) cultures were positive in less than half of cases $[4,5]$.

Received 11/11/2019

Review began 11/11/2019 Review ended 01/11/2020 Published 01/13/2020

\section{() Copyright 2020}

Frade et al. This is an open access article distributed under the terms of the Creative Commons Attribution License CC-BY 3.0., which permits unrestricted use, distribution, and reproduction in any medium, provided the original author and source are credited.
Neuroimaging (CT or MRI) may show focal lesions and/or hydrocephalus.

Although the management of most abscesses requires surgical drainage, antibiotic therapy alone may be successful in treating listerial abscess. The preferred antilisterial agent is ampicillin, sometimes combined with aminoglycosides. If the patient has penicillin hypersensitivity, trimethoprim/sulfamethoxazole is a reasonable alternative [3]. The condition may recur after successful treatment, with or without surgical intervention.

Prognosis may vary according to the presence of risk factors, particularly immunosuppression. Neurological sequelae are common, which depends on the severity and location of the lesion. Mortality is higher than in patients with nonlisterial abscess, but there seems to be no significant difference in mortality between patients with single or multiple listerial abscesses, neither between patients treated with monotherapy or combination therapy $[6,7]$.

\section{Case Presentation}

A 74-year-old female presented to an outside hospital $(\mathrm{OH})$ after a four-day history of headache and a recent fall. Her past medical history was significant for migraines, post-traumatic seizure disorder treated with levetiracetam, coronary artery disease, and supraventricular tachycardia managed with amiodarone and diltiazem. There was no history of alcohol or recreational drug use, but the patient was a chronic smoker. The patient had no focal neurological deficits on presentation to the OH, consistent with a CT head (CTH). Although the patient showed no abnormalities on admission to $\mathrm{OH}$, she developed left-sided hemiparesis over the course of that day. A second CTH performed on the afternoon of the admission was consistent with right basal ganglia hypodensity, so the patient was started on single antiplatelet therapy. Further imaging assessment with MRI showed increased T2 and fluid -attenuated inversion recovery (FLAIR) signals 


\section{Cureus}

involving a subcortical right hemisphere lesion suggestive of glioma, which motivated a transfer to our hospital.

On admission to our hospital, patient was afebrile, eupneic, alert and oriented, with neurological examination revealing paresthesia and mild weakness (4/5) on left upper and lower extremities. Cranial nerve examination and plantar response testing showed no abnormalities. Laboratory examinations initially showed iron-deficiency anemia and mild hyponatremia (Table 1). A repeat brain MRI without contrast showed similar findings to previous imaging, i.e. T2/FLAIR hyperintense lesions in the right basal frontal and temporal lobes, thalamus, and brainstem (Figure 1).

\begin{tabular}{|c|c|c|c|}
\hline Hematologic profile & Value & Metabolic panel & Value \\
\hline Hemoglobin (g/dL) & 9.8 & Sodium (mmol/L) & 131 \\
\hline Hematocrit (\%) & 31.2 & Potassium (mmol/L) & 4.1 \\
\hline Platelets $(1,000 / \mu \mathrm{L})$ & 347 & Chloride (mmol/L) & 96 \\
\hline WBCs $(1,000 / \mu \mathrm{L})$ & 5.38 & Bicarbonate (mmol/L) & 22 \\
\hline$\%$ Neutrophils & $80.9 \%$ & BUN (mg/dL) & 16 \\
\hline$\%$ Immature granulocytes & $0.7 \%$ & Creatinine (mg/dL) & 0.4 \\
\hline \% Lymphocytes & $14.5 \%$ & Glucose (mg/dL) & 156 \\
\hline \% Monocytes & $3.7 \%$ & Calcium (mg/dL) & 8.3 \\
\hline \% Eosinophils & $0.0 \%$ & Magnesium (mg/dL) & 2.1 \\
\hline Serum iron (mcg/dL) & 17 & ALP (u/L) & 65 \\
\hline TIBC (mcg/dL) & 365 & AST (u/L) & 18 \\
\hline Ferritin (ng/mL) & 23.9 & ALT (u/L) & 18 \\
\hline
\end{tabular}

\section{TABLE 1: Laboratory findings on admission}

WBC, white blood cell; TIBC, total iron-binding capacity; BUN, blood urea nitrogen; ALP, alkaline phosphatase; AST, aspartate aminotransferase; $\mathrm{ALT}$, alanine aminotransferase
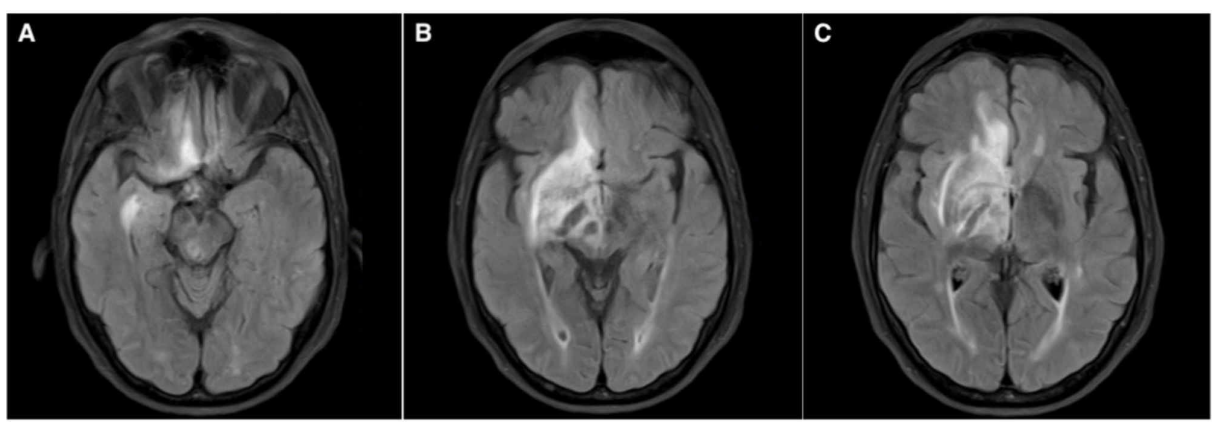

\section{FIGURE 1: Brain MRI on admission}

FLAIR MRI sequence on axial plane showing focal hyperintensities in the midbrain (A), basal frontal and temporal lobes $(A, B)$, thalamus and basal ganglia $(C)$

FLAIR, fluid attenuated inversion recovery

On hospital day 5, the patient underwent stereotactic biopsy of a lesion located in the right frontal lobe, followed by high-volume lumbar puncture. After the procedure, severe hemiparesis (1/5) and a first episode of fever $\left(38.4^{\circ} \mathrm{C}\right)$ were noted, and the following labs on hospital day 6 revealed leukocytosis $(12,580 / \mu \mathrm{L})$ with neutrophilic predominance (84\%). CSF analysis revealed elevated protein and lactate, as well as mild lymphocytic pleocytosis (Table 2), while biopsy revealed signs of acute inflammation and abscess. Followup MRI with contrast revealed multiple ring enhancing lesions in right basal frontal and temporal lobes, 


\section{Cureus}

thalamus, and midbrain (Figure 2). Under the suspicion of septic metastases, empiric treatment was initiated with vancomycin, cefepime, and dexamethasone. After a CSF BioFire ${ }^{\circledR}$ meningitis/encephalitis (ME) panel (BioFire Diagnostics, a bioMérieux Company, Salt Lake City, UT) returned positive for Listeria, antibiotics were switched to ampicillin $12 \mathrm{~g} /$ day and metronidazole $1.5 \mathrm{~g} /$ day. Although the CSF culture was negative, a blood culture was positive for Listeria sensitive to ampicillin and sulfamethoxazole-trimethoprim (Table 3), so metronidazole was discontinued. Seven days after biopsy, culture returned positive for gram-positive organisms consistent with Listeria (Figure 3).

\begin{tabular}{|c|c|c|c|}
\hline & HD 5 & HD 8 & HD 13 \\
\hline Leukocytes $(/ \mu \mathrm{L})$ & 41 & 435 & 159 \\
\hline Neutrophils (\%) & - & 22 & 3 \\
\hline Lymphocytes (\%) & 100 & 78 & 97 \\
\hline Protein (mg/dL) & 65 & 202 & 82 \\
\hline Glucose (mg/dL) & 51 & 83 & 84 \\
\hline Lactate $(\mathrm{mg} / \mathrm{dL})$ & & 66.6 & \\
\hline
\end{tabular}

\section{TABLE 2: Cerebrospinal fluid analysis}

$\mathrm{HD}$, hospital day

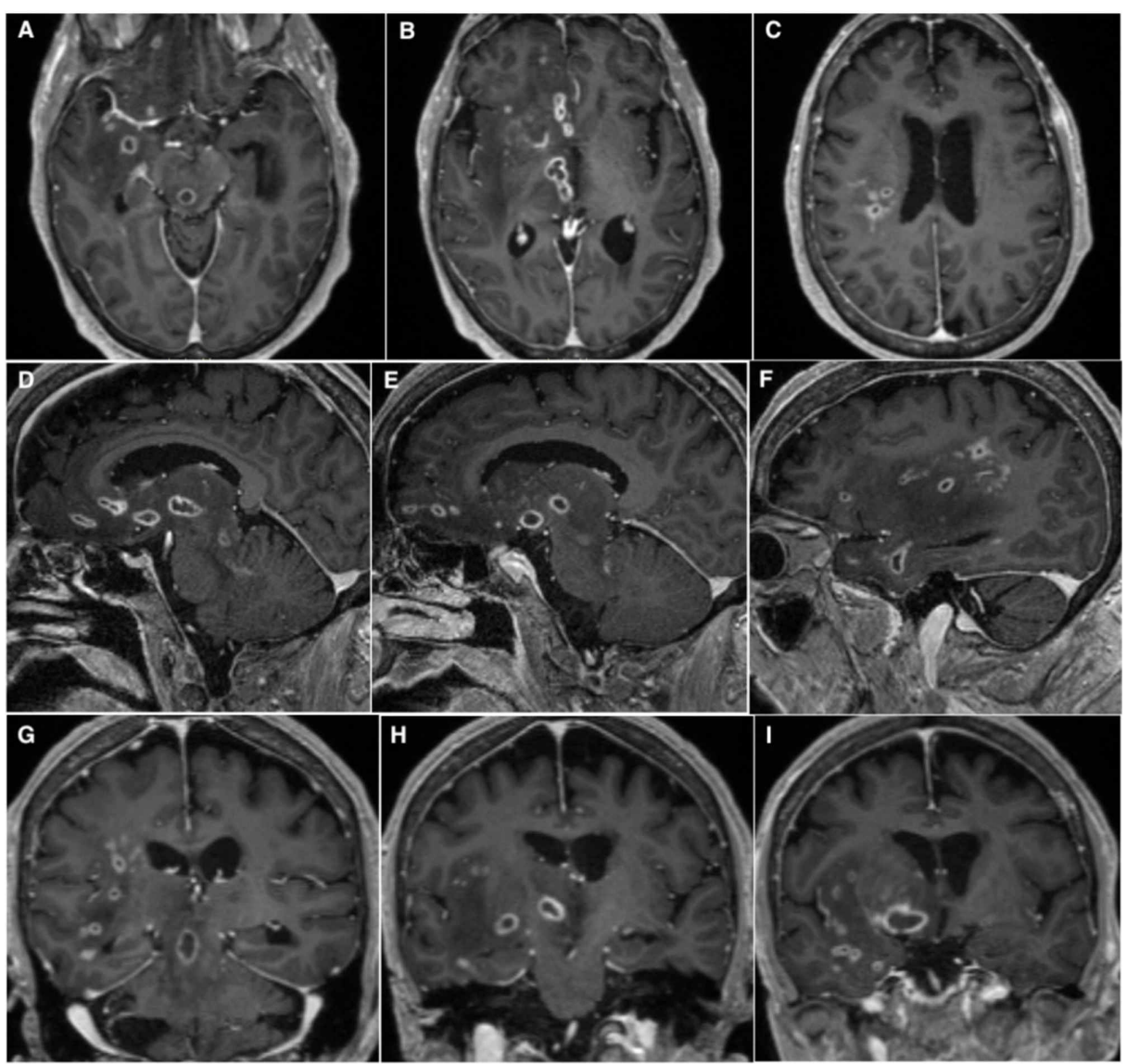

\section{FIGURE 2: Contrast-enhanced MRI}

Contrast-enhanced MRI T1 sequence on axial (A-C), sagittal (D-F), and coronal (G-I) planes showing unilateral ring-enhancing lesions in the midbrain, thalamus, and frontotemporal subcortical regions. 


\section{Cureus}

\begin{tabular}{|c|c|c|c|c|c|}
\hline & HD 5 & HD 6 & HD 7 & HD 8 & HD 13 \\
\hline Blood culture & + & - & - & & \\
\hline CSF culture & - & & & - & - \\
\hline CSF BioFire $\AA$ ME panel & + & & & & \\
\hline Brain tissue culture & Gram + cocci & & & & \\
\hline
\end{tabular}

\section{TABLE 3: Cultures and BioFire ${ }^{\circledR}$ meningitis/encephalitis (ME) panel}

HD, hospital day; CSF, cerebrospinal fluid

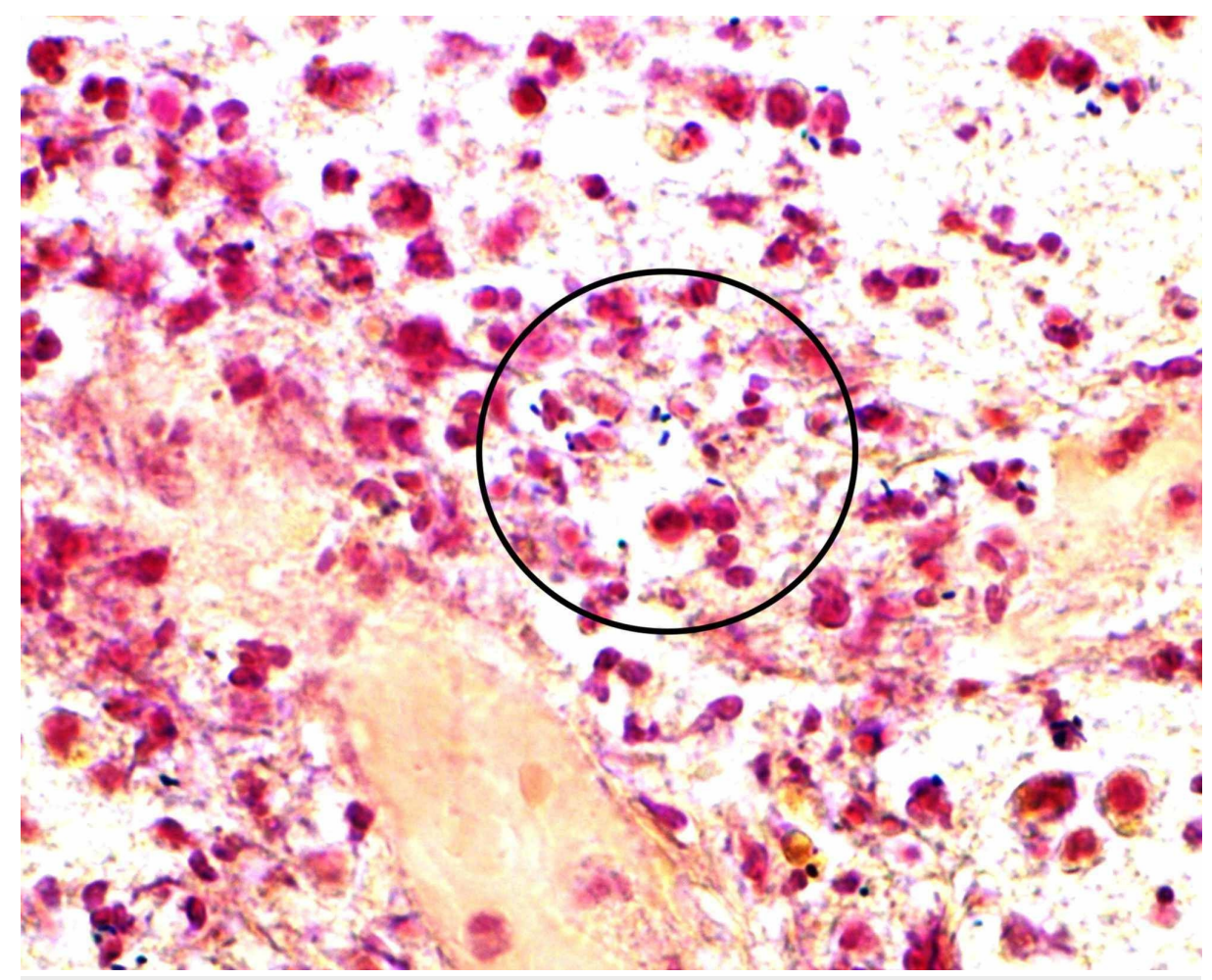

FIGURE 3: Gram stain of brain biopsy specimen

Brain biopsy sample with $\mathrm{X} 60$ magnification showing gram-positive rods

On hospital day 12, the patient developed a lumbar rash consistent with zoster, but skin swab test later revealed herpes simplex virus 2 (HSV-2). A repeat CSF analysis on hospital day 13, eight days after antibiotics were started, was negative for Listeria but positive for HSV-2, consistent with the concurrent skin biopsy findings, so acyclovir was added to the regimen. Due to the concern for immunodeficiency, the patient was tested for HIV, human T-lymphotropic virus, syphilis, viral and autoimmune

hepatitis, hypogammaglobulinemia, and hypocomplementemia, but all the investigations came out to be negative. Flow cytometry revealed a CD4 count of 253 cells/ $/ \mathrm{ll}$. CT and MRI abdomen done for hidden neoplasms revealed hepatic cysts, and serum biomarker screening showed elevated serum carcinoembryonic antigen and cancer antigen 125 .

At the time of discharge, on hospital day 22, patient was conscious and oriented, with neurological examination remarkable only for severe left-sided weakness (1/5) attributed to initial progression of the infection and surgical manipulation. She was transferred to a skilled nursing facility with instructions to complete antibiotic regimen for a total of six weeks, and with scheduled follow-up visits for further assessment of her hepatic condition.

\section{Discussion}


Listeria is a facultative intracellular bacterium mostly associated with foodborne illness, but it also can cause CNS infection, neurolisteriosis, particularly in the setting of impaired cell-mediated immunity. Neurolisteriosis commonly affects the meninges, but it may also affect brain parenchyma. Subcortical areas, thalamus, pons, and medulla are usual targets [8]. In this patient, there were multiple ring-enhancing lesions suggestive of abscesses in right frontotemporal subcortical areas, thalamus, and midbrain. More than one foci have been described in only $25 \%$ of patients with listerial abscesses [6].

This patient had a gradual course of worsening headache and focal findings, the left-sided weakness, and paresthesia, all of which are congruent to a CNS abscess rather than meningitis. There were no fever, nausea, vomiting, or diarrhea reported before or after admission to our hospital. Blood tests revealed mild leukocytosis, which was aligned with the previous reports $[9,10]$. CSF analysis showed elevated levels of lactate, which has been reported as a useful diagnostic marker to differentiate between viral CNS infections and Listeria or other bacterial infections [11].

In microbiology studies, we found positive blood culture associated with negative CSF culture, consistent with the previous findings [7]. Our CSF was further analyzed with a nucleic acid amplification test (NAAT), a BioFire ${ }^{\circledR}$ ME panel, which was positive for Listeria DNA, and tailored our empiric antibiotic regimen to cover Listeria. This suggests an important role for NAAT, as a faster and considerably sensitive alternative for ruling in Listeria before culture results are available, potentially improving morbidity and mortality.

Abscess is particularly rare among neurolisteriosis presentations and is often related to immunosuppression. In a review of 39 cases of Listeria abscesses, $85 \%$ of patients were immunocompromised by HIV infection, lymphoma, leukemia, or conditions requiring chronic immunosuppressive therapy [4]. Our patient initially presented with no evidence of immunosuppressive treatment or condition, but workup revealed hepatic lesions and elevated tumor markers concerning for malignancy. These findings could represent a malignancy contributing in some degree to immune suppression, although there was no confirmatory evidence by the time the patient was discharged. Also, during her inpatient course, she developed skin lesions secondary to HSV-2 reactivation. Even though herpesvirus reactivations suggest immune suppression, they may also be related to normal aging [12].

Our patient showed clinical improvement after empiric and sensitivity-guided antibiotic regimens for Listeria, but still retained left-sided weakness by the time of discharge, likely secondary to both abscess burden and brain surgery. Timely initiation of empiric antibiotics has been associated with better clinical outcome [13]. For better diagnostic accuracy and antibiotic coverage, an empiric regimen should be started shortly after the aspiration of one abscess has been performed, and should be able to cover staphylococci, streptococci, strict anaerobes, and Enterobacteriaceae [14].

Multiple abscesses by Listeria are a rare presentation of neurolisteriosis and have been mostly reported in immunosuppressed adult or elderly patients [6]. Although there was no confirmatory evidence of immune suppression in this patient by the time she was discharged, there were scheduled follow-up appointments for further assessment of the recently found hepatic cystic lesions.

\section{Conclusions}

We reported an atypical presentation of CNS listerial infection in a previously immunocompetent patient. Despite initially non-focal symptoms, further testing revealed multiple listerial abscesses. After initiation of antibiotics, this patient had a good response and a favorable short-term outcome. Although rare in immunocompetent patients, it is important to consider Listeria in the differential diagnosis of single or multiple brain abscesses.

\section{Additional Information \\ Disclosures}

Human subjects: Consent was obtained by all participants in this study. MU Institutional Review Board issued approval 2016974. Conflicts of interest: In compliance with the ICMJE uniform disclosure form, all authors declare the following: Payment/services info: All authors have declared that no financial support was received from any organization for the submitted work. Financial relationships: All authors have declared that they have no financial relationships at present or within the previous three years with any organizations that might have an interest in the submitted work. Other relationships: All authors have declared that there are no other relationships or activities that could appear to have influenced the submitted work.

\section{References}

1. Scallan E, Hoekstra RM, Angulo FJ, et al.: Foodborne illness acquired in the United States: major pathogens . Emerg Infect Dis. 2011, 17:7-15. 10.3201/eid1701.P11101

2. Schuchat A, Robinson K, Wenger JD, et al.: Bacterial meningitis in the United States in 1995 . N Engl J Med. 1997, 337:970-976. 10.1056/NEJM199710023371404

3. Clauss HE, Lorber B: Central nervous system infection with Listeria monocytogenes. Curr Infect Dis Rep. 


\section{Cureus}

2008, 10:300-306. 10.1007/s11908-008-0049-0

4. Eckburg PB, Montoya JG, Vosti KL: Brain abscess due to Listeria monocytogenes. Medicine (Baltimore). 2001, 80:223-235. 10.1097/00005792-200107000-00001

5. Dee R, Lorber B: Brain abscess due to Listeria monocytogenes: case report and literature review . Rev Infect Dis. 1986, 8:968-977. 10.1093/clinids/8.6.968

6. Cone LA, Leung MM, Byrd RG, Annunziata GM, Lam RY, Herman BK: Multiple cerebral abscesses because of Listeria monocytogenes: three case reports and a literature review of supratentorial listerial brain abscess (es). Surg Neurol. 2003, 59:320-328. 10.1016/S0090-3019(03)00056-9

7. Tiri B, Priante G, Saraca LM, Martella LA, Cappanera S, Francisci D: Listeria monocytogenes brain abscess: controversial issues for the treatment-two cases and literature review. Case Rep Infect Dis. 2018, 2018:1-9. $10.1155 / 2018 / 6549496$

8. Disson O, Lecuit M: Targeting of the central nervous system by Listeria monocytogenes . Virulence. 2012, 3:213-221. 10.4161/viru. 19586

9. Khan S, Kumar A, Kale S, Kurkure N, Nair G, Dinesh K: Multiple cortical brain abscesses due to Listeria monocytogenes in an immunocompetent patient. Trop Doct. 2018, 48:160-163. 10.1177/0049475517728670

10. Soares-Fernandes JP, Beleza P, Cerqueira JJ, et al.: Simultaneous supratentorial and brainstem abscesses due to Listeria monocytogenes. J Neuroradiol. 2008, 35:173-176. 10.1016/j.neurad.2007.07.009

11. Cunha BA, Fatehpuria R, Eisenstein LE: Listeria monocytogenes encephalitis mimicking herpes simplex virus encephalitis: the differential diagnostic importance of cerebrospinal fluid lactic acid levels. Hear Lung J Acute Crit Care. 2007, 36:226-231. 10.1016/j.hrtlng.2007.01.001

12. Grinde B: Herpesviruses: latency and reactivation - viral strategies and host response . J Oral Microbiol. 2013, 5:1-9. 10.3402/jom.v5i0.22766

13. DeJesus-Alvelo I, Merenda A: A case report of Listeria monocytogenes abscesses presenting as cortically predominant ring-enhancing lesions. Case Rep Neurol. 2015, 7:105-109. 10.1159/000430434

14. Sonneville R, Ruimy R, Benzonana N, et al.: An update on bacterial brain abscess in immunocompetent patients. Clin Microbiol Infect. 2017, 23:614-620. 10.1016/j.cmi.2017.05.004 